\title{
Selection between aztreonam and cephalosporins for treatment of infections with pseudomonads needs more caution
}

\section{Bhoj R Singh}

Division of Epidemiology, ICARIndian Veterinary Research Institute, Izatnagar, India
Correspondence: Bhoj R Singh Division of Epidemiology, ICAR-Indian Veterinary Research Institute, Izatnagar 243122, India

Email brs 1762@gmail.com
This article was published in the following Dove Medical Press journal: Infection and Drug Resistance

\section{Dear editor}

In the recently published study ${ }^{1}$ to evaluate the use of aztreonam as an active empiric therapy against subsequent culture of Pseudomonas aeruginosa, empiric therapy failure using aztreonam is reported more common than on using $\beta$-lactam antibiotics in patients suffering $P$. aeruginosa infection. Though the study is interesting and revealing important findings regarding antibiotic use for treatment of $P$. aeruginosa infection, it should be accepted with caution as suggested by the authors ${ }^{1}$ repeatedly due to limited number of cases. In our observations on $P$. aeruginosa (95) and other pseudomonads (40) isolates from veterinary clinical cases we found that instead of generalizing the lesser efficacy of aztreonam in-depth studies are required. Although insignificant, aztreonam inhibited more numbers of extended spectrum $\beta$-lactamase (ESBL) producing (57) P. aeruginosa strains (56.1\%) than most of the $\beta$-lactams including cefotaxime, ceftriaxone and piperacillin (53.3\%). However, on non-ESBL producing (37) strains aztreonam inhibited $42.1 \%$ isolates, much less than cefepime (68\%), ceftriaxone $(50 \%)$ and piperacillin + tazobactam (61.1\%). Therefore, it is suggested to use the two classes of antibiotics (aztreonam and $\beta$-lactams) judiciously based on antibiotic stewardship principle $^{1}$ instead of following some general rule for infections with pseudomonads.

In the analysis, antibiotic sensitivity patterns, available in clinical epidemiology laboratory of the Institute, of the 82 ESBL producers pseudomonads including $P$. aeruginosa (57), P. alcaligenes (1), P. fluorescens (7), P. paucimobilis (8), P. pseudoalcaligenes (5), P. stutzeri (3), P. testosteronii (1) and 53 ESBL negative pseudomonads including P. aeruginosa (38), P. alcaligenes (1), P. diminuta (1), P. fluorescens (6), P. paucimobilis (2), P. pseudoalcaligenes (3), P. stutzeri (1), P. vesicularis (1) were included. All the isolates were associated with one or other clinical condition in animals and were tested for antibiotic sensitivity pattern using standard disc diffusion assay. ${ }^{2}$

The analysis of the data (Table 1) for sensitivity of $P$. aeruginosa included in the study for aztreonam, carbapenems (meropenem, imipenem, ertapenem), cefepime, cefotaxime, ceftriaxone, colistin, gentamicin, piperacillin + Tazobactam, tetracycline and tigecycline revealed ESBL negative $P$. aeruginosa $(\mathrm{PA})$ isolates were more often (though statistically insignificant, $P>0.05$ ) resistant $(57.9 \%)$ to aztreonam than the ESBL positive isolates (43.9\%). However, significantly more number of the ESBL negative $P$. aeruginosa isolates resisted gentamicin $(P<0.001)$ than ESBL positive 
Table I Antibiotic sensitivity pattern of Pseudomonas aeruginosa and other pseudomonads isolated from clinical infections in animals

\begin{tabular}{|c|c|c|c|c|c|c|}
\hline \multirow[t]{2}{*}{ Antibiotics tested } & \multicolumn{2}{|c|}{ P. aeruginosa $(\mathrm{N}=95)$} & \multicolumn{2}{|c|}{ Other pseudomonads $(\mathrm{N}=40)$} & \multicolumn{2}{|l|}{ Total $(\mathbf{N}=135)$} \\
\hline & ESBL-ve (38) & ESBL+ve (57) & ESBL-ve (15) & ESBL+ve (25) & ESBL-ve (53) & ESBL+ve (82) \\
\hline Aztreonam & 42.1 & 56.1 & 46.7 & 64.0 & 43.4 & 58.5 \\
\hline Carbapenems & 57.9 & 66.7 & 50.0 & 33.3 & 64.2 & 67.1 \\
\hline Cefepime & 68.0 & 90.0 & 40.0 & 60.0 & 62.9 & 72.4 \\
\hline Cefotaxime & 31.6 & 42.1 & 66.7 & 64.0 & 34.0 & 47.6 \\
\hline Ceftriaxone & 50.0 & 49.1 & 66.7 & 84.0 & 54.7 & 53.7 \\
\hline Colistin & 89.5 & 93.0 & 80.0 & 68.0 & 83.0 & 90.2 \\
\hline Gentamicin & 68.4 & 94.7 & 93.3 & 88.0 & 75.5 & 92.7 \\
\hline Piperacillin + Tazobactam & 61.1 & 53.3 & 64.3 & 78.9 & 62.0 & 60.9 \\
\hline Tetracycline & 18.4 & 21.1 & 46.7 & 60.0 & 26.4 & 32.9 \\
\hline Tigecycline & 28.9 & 42.1 & 80.0 & 72.0 & 43.4 & 51.2 \\
\hline
\end{tabular}

Abbreviations: ESBL-ve, extended spectrum $\beta$-lactamase negative; ESBL+ve, extended spectrum $\beta$-lactamase positive.

isolates. The ESBL negative $P$. aeruginosa isolates were significantly more commonly resistant to aztreonam than to cefepime $(P=0.04)$, colistin $(P<0.001)$ and gentamicin $(P=0.02)$ but less often than to tetracycline $(P=0.025)$. The ESBL positive $P$. aeruginosa isolates were significantly $(P$ $<0.001)$ more often resistant to aztreonam than to colistin and gentamicin but less than to tetracycline. The ESBL and non-ESBL pseudomonads other than P. aeruginosa (NPA) had not differed significantly $(P>0.05)$ in their sensitivity to any of the antibiotics, however, aztreonam inhibited $64 \%$ ESBL producers and only $46.7 \%$ of ESBL negative isolates. Though for most of the antibiotics, including aztreonam and cephalosporins, sensitivity of $P$. aeruginosa and NPA had not differed significantly $(P>0.05)$, NPAs were significantly more often resistant to cefepime $(P<0.001)$, colistin $(P=0.02)$, but less often $(P<0.001)$ to tetracycline and tigecycline.
The analysis on antibiotic sensitivity patterns of $P$. aeruginosa and NPA isolates from veterinary clinical cases indicated need of antibiotic sensitivity assay for judicious use of antibiotics in therapy ie, need to reinforce antimicrobial stewardship principles. ${ }^{1}$

\section{Disclosure}

The author reports no conflicts of interest in this communication.

\section{References}

1. Hogan M, Bridgeman MB, Min GH, Dixit D, Bridgeman PJ, Narayanan $\mathrm{N}$. Effectiveness of empiric aztreonam compared to other beta-lactams for treatment of Pseudomonas aeruginosa infections. Infect Drug Resist. 2018;11:1975-1981.

2. CLSI. Performance Standards for Antimicrobial Disk Susceptibility Tests. 24th informational supplement M100-S24 and M11-A8. Wayne, PA, USA: Clinical and Laboratory Standards Institute; 2014. 


\section{Authors' reply}

\section{Response to Letter to the Editor regarding publication, Effectiveness of empiric aztreonam compared to other beta-lactams for treatment of Pseudomonas aeruginosa infections}

\author{
Michael Hogan' \\ Mary Barna Bridgeman' \\ Gee Hee Min' \\ Deepali Dixit ${ }^{\prime}$ \\ Patrick J Bridgeman' \\ Navaneeth Narayanan ${ }^{1,2}$ \\ 'Department of Pharmacy Practice and Administration, Ernest \\ Mario School of Pharmacy, Rutgers University, Piscataway, NJ, USA; \\ ${ }^{2}$ Division of Infectious Diseases, Department of Medicine, Rutgers, \\ Robert Wood Johnson Medical School, New Brunswick, NJ, USA \\ Correspondence: Mary Barna Bridgeman \\ Department of Pharmacy Practice and Administration, Ernest Mario \\ School of Pharmacy, Rutgers University, I 60 Frelinghuysen Road, \\ Piscataway, NJ 08854, USA \\ $\mathrm{Tel}+\mathrm{I} 8484456815$ \\ Email Mary.bridgeman@pharmacy.rutgers.edu
}

\section{Dear editor}

We appreciate the author of this letter reading our article with such great interest. We believe, however, in this response that there is limited application to the context and overall content of our clinical study of human patients. First, the data cited in this response are from veterinary clinical cases. Though general principles of understanding of antibiotic sensitivity testing and resistance mechanisms apply regardless of species, there are major differences that impede reasonable comparisons between the assertions in this letter and findings of our original study. This includes the choice of antibiotics used, at least for sensitivity testing for Pseudomonas in animals, as cited by Singh. Antibiotics such as cefotaxime, ceftriaxone, tetracycline, and tigecycline are never clinically utilized or tested for susceptibility against Pseudomonas aeruginosa (P. aeruginosa) in the treatment of human infections. It is universally taught to clinicians that these antibiotics lack clinically relevant activity against $P$. aeruginosa and therefore should not be used as empiric therapy if there is potential for infection with $P$. aeruginosa; similarly, these agents are not tested to assess as an option for definitive therapy based on a lack of clinical efficacy. ${ }^{1,2}$ Second, Singh cited data by stratifying $P$. aeruginosa by extended-spectrum beta-lactamase (ESBL) production and compared aztreonam susceptibility to ceftriaxone, cefotaxime, and piperacillin. At least in the US, genotypic testing for ESBL production in $P$. aeruginosa is not routinely performed in the clinical setting and as mentioned previously, many of the antibiotics mentioned (eg, cefotaxime and ceftriaxone) would never be tested against $P$. aeruginosa regardless of suspicion of ESBL production. The differential susceptibility by ESBL production, therefore, has limited application. Additionally, in the US where our study takes place, plasmid-mediated ESBL production by $P$. aeruginosa is not common while AmpC beta-lactamase production is the primary beta-lactamase resistance mechanism found in $P$. aeruginos $a{ }^{3}$ Third, and finally the primary objective and underlying context of our study is not readily considered. We sought to assess the utility of aztreonam as an empiric agent (prior to any culture and susceptibility results) against $P$. aeruginosa in human clinical cases as compared to other antipseudomonal beta-lactams (eg, cefepime, piperacillin/tazobactam and not agents such as ceftriaxone). The final point by Singh notes the need for antibiotic sensitivity testing for judicious use of antibiotics, which we believe is true for most clinical cases of infection in general but overlooks the message of empiric therapy selection, which is what our study aimed to evaluate.

\section{Disclosure}

The authors report no conflicts of interest in this communication.

\section{References}

1. Gilbert DN, Chambers HF, Eliopoulos GM, Saag MS, Pavia AT, editors Sanford Guide to Antimicrobial Therapy 2018. 48th ed. Sperryville, VA: Antimicrobial Therapy, Inc.; 2018.

2. CLSI. Performance Standards for Antimicrobial Susceptibility Testing. 28th ed. CLSI supplement M100. Wayne, PA: Clinical and Laboratory Standards Institute; 2018.

3. Nguyen L, Garcia J, Gruenberg K, MacDougall C. Multidrug-resistant pseudomonas infections: hard to treat, but hope on the horizon? Curr Infect Dis Rep. 2018;20(8):23. 
Dove Medical Press encourages responsible, free and frank academic debate. The content of the Infection and Drug Resistance 'letters to the editor' section does not necessarily represent the views of Dove Medical Press, its officers, agents, employees, related entities or the Infection and Drug Resistance editors. While all reasonable steps have been taken to confirm the content of each letter, Dove Medical Press accepts no liability in respect of the content of any letter, nor is it responsible for the content and accuracy of any letter to the editor.

Infection and Drug Resistance

Dovepress

\section{Publish your work in this journal}

Infection and Drug Resistance is an international, peer-reviewed openaccess journal that focuses on the optimal treatment of infection (bacterial, fungal and viral) and the development and institution of preventive strategies to minimize the development and spread of resistance. The journal is specifically concerned with the epidemiology of antibiotic resistance and the mechanisms of resistance development and diffusion in both hospitals and the community. The manuscript management system is completely online and includes a very quick and fair peerreview system, which is all easy to use. Visit http://www.dovepress.com/ testimonials.php to read real quotes from published authors.

Submit your manuscript here: https://www.dovepress.com/infection-and-drug-resistance-journal 